\title{
Dual Use of Carbon Nanotube Selective Coatings in Evacuated Tube Solar Collectors
}

\author{
Patricia M. Martinez ${ }^{1,5}$ Vladimir A. Pozdin, ${ }^{2}$ Alexios Papadimitratos, ${ }^{1,2}$ William Holmes, ${ }^{2}$ \\ Fatemeh Hassanipour, ${ }^{3}$ Anvar A. Zakhidov ${ }^{1,4^{*}}$
}

\begin{abstract}
Continuous carbon nanotube sheets spun from the side of vertically aligned forests were investigated as solar selective coatings in evacuated tube collectors for solar thermal applications and for Joule heating in the absence of solar radiation. Forests grown by chemical vapor deposition at various synthesis temperatures produced sheets with tunable thermal and optical properties. The sheets were laminated on miniature and regular size evacuated tube collectors and tested at $100 \mathrm{~mW} / \mathrm{cm}^{2} \mathrm{AM} 1.5$ solar illumination under high vacuum. It was found that 10 layers of carbon nanotube sheets with medium sheet areal density produced the best solar selective coating. Interestingly, carbon nanotube sheet densification with organic solvents slightly increased the reflectance and significantly decreased the emissivity; improving the
\end{abstract}


thermal transport even further. By taking advantage of the Joule heating effect, carbon nanotube sheets provide fast and efficient heating rates on demand, which can be useful in solar water heating systems when solar radiation is insufficient. Compared with commercial coatings, carbon nanotube sheets were found to have higher absorption and lower reflection in the visible and near UV region of the solar spectrum, making them ideal solar selective coatings for evacuated tube collectors even in overcast weather.

\section{Introduction}

At the current consumption rates fossil fuel, natural gas and coal represented 46, 58 and 150 years of remaining energy provisions in 2010, respectively [1]. The limited fossil fuel reserves and their negative environmental consequences have led to greater deployment of renewable energies with cleaner and unlimited resources that are not geographically restricted. According to the International Energy Agency (IEA), in 2014 the worldwide energy consumption was 12.3 TW from which $15.5 \%$ came from renewable energies. Of these $15.5 \%$, $12.2 \%$ represents biofuels and $3.3 \%$ is attributed to solar, geothermal, wind and heat energy conversion [2]. Of the available renewable energies, solar has the greatest potential to surpass the worldwide energy consumption going into the future. If the entire surface area of the planet exposed to sunlight could be devoted to this goal with excellent harvesting and storage capacity, 124 PW of energy could be generated or approximately 10,000 times the worldwide energy consumption in 2013. Properly managed, solar energy could provide enough energy to exceed the total estimated lifetime of fossil fuel resources in a single year [1].

Currently, solar energy can be converted into electrical energy via photovoltaic technology or into thermal energy by utilizing solar thermal collectors, specifically solar water 
heaters (SWHs). In a SWH system, incident solar energy is converted to heat by a solar collector and transferred through a transfer medium, such as water, to a heat storage tank. The most important part of a SWH is the solar selective coating (SSC); it is the active component which converts the incident solar radiation into heat, and its energy conversion efficiency is, therefore, integral to the utility of the device as a whole [3]. For optimal solar thermal energy conversion, an SSC needs to discriminate between wavelengths from the solar spectrum and the black body radiation. High absorption and low reflectance in the visible region, as well as, high reflectance in the infrared region and low thermal emissivity are needed at a corresponding temperature[47]. For an operational temperature of $100^{\circ} \mathrm{C}$, the optimal crossover wavelength is $1-2 \mu \mathrm{m}$ [4]. Carbon-based materials, such as graphene and carbon nanotubes (CNT), have gained enormous attention over the past decade due to their excellent optical [8,9], thermal [10,11], electrical and mechanical properties [12] and have been successfully implemented as SSCs. Graphene has been coated on top of commercially available flat plate collectors in the form of nanofluid [13], as transparent heaters on top of a polyethylene terephthalate (PET) with interlayer P-doped graphene films [14] or as porous sponges that generate steam by harvesting solar energy with $80 \%$ efficiency [11]. Single and multi-walled CNTs have been reported to be nearly-perfect optical absorbers with ultra-low theoretical reflection of $0.01-0.10 \%$ in the visible spectrum [15], making them ideal SSCs. Solution of CNT has been successfully used as selective coatings; Abendroth et al. (2016), deposited single-walled CNT solutions on top of a quartz substrate by ultrasonic spraying and found that film thickness influenced the optical properties. Others have demonstrated that multi-walled CNTs electrophoretic deposited on top of aluminum substrates can achieved high absorbance (90\%) and low thermal emissivity (0.13) with $1.7 \mu \mathrm{m}$ thick CNT film [16]. With CNT solutions the observed trend is that high absorbance and low reflectance in 
the visible spectrum and high reflectance in the infrared region can be achieved with thicker films, but at the cost of increased thermal emissivity [16].

The use of aligned CNT forest has also been reported as SSC. For example, Yang et al. (2008), achieved a total reflectance of $0.045 \%$ by using long, low-density and well-aligned forest with deep apertures [15]. Cao et al. (2002), grew CNT forests on gold coated quartz with almost perfect absorption in the visible and IR regions for CNT lengths $>10 \mu \mathrm{m}$, however, for lengths less than $5 \mu \mathrm{m}$, the reflectance increased and absorptance decreased [17]. Other groups have created CNT-tandem absorbers made of $\mathrm{Ti}|\mathrm{AlTiO}| \mathrm{CoO} \mid \mathrm{CNT}$ forest that act as a black body absorber or as an SSC by varying the forest thickness[18].

Although CNT forest and solutions have shown interesting results as SSCs, they are not suitable for use in SWHs. In many cases, the SSC required the deposition of a reflective surface made of expensive metals, such as gold, titanium or aluminum on top of quartz or stainless steel by sputtering techniques[16-18]. Thus, the production at large scale is limited by cost and deposition technique. CNT forest's delicate morphology and difficulty in growing in larger areas make it almost impossible to use CNT forests at large scales in SWH. CNT solutions could be a more appropriate SSC material for SWHs; however, a significant amount of CNTs is necessary to coat the collector and could be difficult to apply precisely and in an oriented way to specific areas.

To date, aligned CNT sheets have not been tested as SSCs. These sheets can be continuously laminated on top of large surfaces with excellent adhesion and can be stacked on top of one another to produce enhanced optical (transmittance, reflectivity and absorptance), electrical and thermal properties. Demonstrated in 2005 by Zhang et al., these CNT sheets are dry-spun from a vertically aligned multi-walled CNT forest grown by chemical vapor deposition 
(CVD). From a length $(d)$ of $1 \mathrm{~cm}$ of forest area, $3-12 \mathrm{~m}$ length $(L)$ of continuous CNT sheet can be drawn (with pulling ratio $r=L / d$, dependent on the CVD growth conditions) at a rate of 10 $\mathrm{m} / \mathrm{min}[30]$.

Despite the fact that almost all CNTs are grown by CVD, due to its simple, low temperature and ambient pressure technique [19-23] and the ability to control CNT length, orientation density and morphology [16,20-29], not all forests can be spinnable [21]. Spinnability strategically depends on gas type and flow rates, synthesis temperatures and type of catalytic metal used $[19,21,23,25]$. The main difference between spinnable CNTs and other carbonaceous materials grown by CVD is that the substrate does not form part of the dry-spun sheets $[19,23]$.

When comparing CNT forest and other materials grown by CVD, the catalytic metal, does not undergo a chemical reaction, instead it changes solubility by undergoing phase transitions [30-32]. Thus, CVD reaction temperature oversees the catalytic metal solubility and decomposition of the reaction gases that will diffuse into the supported metal and grow CNTs. Therefore, the chosen substrate does not play an important role in the synthesis (i.e., spinnable forests have been grown on stainless steel and silicon substrates with or without buffer layers, without affecting the spinnability $[19,23,33])$. However, other materials, for example cuprous oxide $\left(\mathrm{Cu}_{2} \mathrm{O}\right)$, a metal-oxide semiconductor with high absorption in the visible region [34], goes through $\mathrm{Cu}$ chemical oxidation inside the CVD chamber and its crystallographic direction is dependent on the type of substrate used: $\mathrm{Cu}_{2} \mathrm{O}$ (111) or (200) for sapphire or $\mathrm{MgO}$ respectively $[34,35]$.

To our knowledge, the use of CNT sheets for an SSC has not been investigated before. Thus, the objective of this work is to explore the solar selectivity of the CNT sheets (optical and 
thermal properties) spun from forests grown at different CVD synthesis temperatures. The best CNT-SSC will be laminated on evacuated tube collectors (ETC) and tested as a Joule heater to generate hot water in the absence of sunlight. An ETC consists of two concentric glass tubes: an inner tube, coated with the SSC material, fused to an external tube at one end. Vacuum is created between the two tubes to minimize convection losses [36]. The heat converted by the SSC is extracted using a heat pipe, a u-tube or by direct water flow [7]. The rounded morphology of the SSC coated tube will allow for a continuous normal incidence angle between the sun rays the CNT-SSC. Thus, constant absorption maxima can be achieved as the sun traverses the sky [3742].

\section{Experimental}

\subsection{CNT synthesis}

Silicon wafers with $50 \mathrm{~nm}$ silicon dioxide and $3 \mathrm{~nm}$ of catalytic iron film deposited by electron-beam evaporation (CHA-50) were used as substrates. To start the CNT synthesis, 3 by $4 \mathrm{~cm}$ substrate pieces were loaded into the CVD reaction chamber, flushed three times with helium before beginning the reaction and maintained under $2400 \mathrm{sccm}$ until the reaction was finished. A three-zone furnace was used to set the synthesis temperature at $700-750^{\circ} \mathrm{C}$ with 30 min ramp time. The synthesis started when $115 \mathrm{sccm}$ of acetylene and $1350 \mathrm{sccm}$ of hydrogen entered the reaction chamber at a set temperature. The actual synthesis time was kept at 10 min and the temperature was varied to produce forests of various heights. Upon completion of the synthesis, reaction gasses were turned off and the system was allowed to cool down to room temperature under helium flow. 
The spinnability of forests was rated from 0 to 5, based on the rating proposed by Huynh et al. (2010). A rating of 0 corresponds to a non-spinnable forest from which no CNT sheet can be spun. 5 indicates that a continuous CNT sheets can be spun from the forest without decreasing its width.

\subsection{Sheet pulling on small and large scale}

CNT solar selective coatings were prepared by pulling CNT sheets from the side of a spinnable forests [30]. A custom-made lamination system (Figure 1a) containing a stepper motor and a linear stage (Olympus belt drive actuator) was used to laminate the CNT sheets with multilayer morphologies on top of small test tubes $(15 \mathrm{~cm}$ length by $2 \mathrm{~cm}$ diameter) and onto a half-meter ETC for thermal and Joule heating testing, respectively. The rotational speed and stage motion were controlled using a custom LabView program with additional input for the width of the CNT sheet. Coatings with precise sheet overlap and multilayer morphologies were produced by controlling the number of CNT sheet layers per pass (Figure 1b). Densification of CNT coating was done after the lamination process by wetting the coating with organic liquids, such as isopropyl alcohol or ethanol, and allowing it to dry at room temperature. This resulted in CNT thickness reduction of $300-400$ times (from an initial $20-30 \mu \mathrm{m}$ aerogel to $50-100 \mathrm{~nm}$ thick sheet) and an improved attachment to the collector surface by Van der Waals forces.
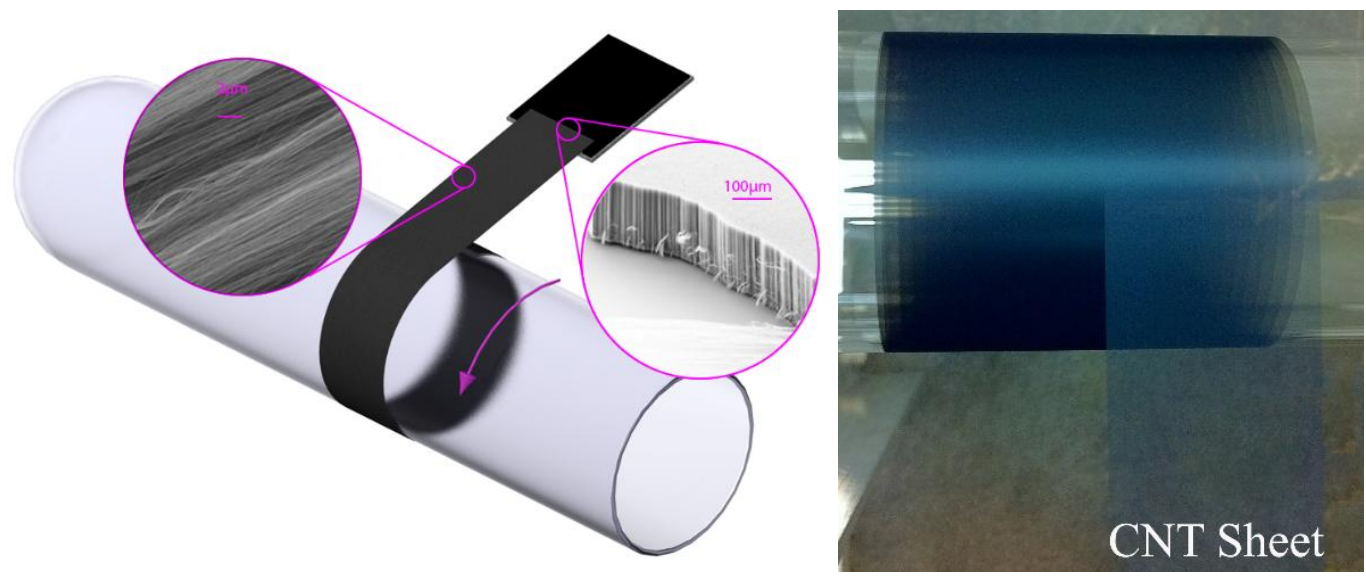
Figure 1. Lamination process to coat tubular collectors with CNT sheets as solar selective coatings. a) CNT aerogel sheets are dry-spun from a vertically aligned forest and onto a collector surface. b) Precise coating structure is achieved with careful control of sheet overlap. Overlap between layers is $1 / 15$ of sheet width.

\subsection{Sheet areal density measurements}

The sheet areal density on one CNT sheet was calculated by dividing the weight of one CNT sheet by its area. The weight of one CNT sheet was calculated by extrapolating the weight of 5, 8, 10 and 15 layers of CNTs stacked on top of each versus the number of CNT sheets. The weight of the sheets was an average of 3 measurements recorded with a microgram accurate scale. To minimize human error, photographs on the dimensions of the sheets (width and length) were obtained by using an optical microscope with a camera attached. The photographs were then analyzed with MatLab to obtain a more accurate estimate of the area.

\subsection{Optical measurements}

Optical properties of carbon nanotube sheets were investigated using Perkin Elmer Lambda 900 UV-Vis/NIR Spectrophotometer. Samples were prepared on quartz substrates. Reflectivity measurements were collected using an integrating sphere attachment. Measurements of reflection, $\% R$, and transmission, $\% T$ were combined to calculate absorption, $\% A$, as $A=1-R-T$. Spectral noise and discontinuity in the UV-Vis data around $860 \mathrm{~nm}$ were due to crossover between light sources and detectors.

Commercial solar collectors obtained for comparative measurements were Apricus Al-N$\mathrm{Cu} / \mathrm{Al}$ and WesTech Al-N SS. Due to sample constraints of the UV-Vis Spectrophotometer, 
commercial collectors were cut into pieces using a diamond saw for measurements. Because of the inherent curvature of the collector, the collected reflectivity measurements of commercial coatings could be, in fact, an underestimation of the total reflectivity due to the imperfect contact with the flat integrating sphere port.

Measurements of carbon nanotube reflectivity in the IR spectrum were collected using a Perkin Elmer Spectrum GX FTIR.

\subsection{SEM and Raman measurements}

Morphology of CNT forests and sheets was investigated using a Zeiss-LEO Model 1530 Variable Pressure Field Effect Scanning Electron Microscope. Jobin Yvon LabRam HR MicroRaman Spectrometer with a $630 \mathrm{~nm}$ excitation laser was used to measure the CNT sheet Raman shift.

\subsection{Emissivity measurements}

Thermal emissivity of the samples, $\varepsilon_{t h}$, at $100^{\circ} \mathrm{C}$ was measured using an FTIR E40 infrared camera. Heat gun was used to heat the samples to $100^{\circ} \mathrm{C}$ as monitored by a surface thermocouple. The emissivity value was obtained by matching the actual sample surface temperature to the reading on the FTIR camera.

\subsection{Testing in High Vacuum system}

Miniature solar collectors were evaluated inside a custom vacuum chamber at high vacuum $\left(10^{-5}\right.$ Torr $)$. This simulated the conditions of completed solar collectors, where the SSC is in vacuum between two concentric glass tubes. Oriel Solar simulator was used to illuminate a section of the SSC through a glass bell jar. The light source was filtered to AM 1.5 and calibrated to $100 \mathrm{~mW} / \mathrm{cm}^{2}$ using a silicon reference diode. Surface thermocouples were mounted inside the miniature collector at two locations: one on the illuminated section and another on the opposite, 
the non-illuminated section. Under illumination, a thermocouple logger (Omega HH309A) recorded the temperature increase due to absorption and transmission of the solar energy.

\subsection{Joule heating}

The completed half-meter collector with CNT composite coating was used to evaluate the performance of Joule heating. To eliminate the effect of the heat pipe on the performance, the inside of the collector tube was directly filled with water $(700 \mathrm{ml})$. A stirring rod was used to agitate the water and ensure accurate thermocouple readings, as fast Joule heating of the CNT coating creates a significant temperature gradient across non-perturbed water. The water temperature was measured by multiple thermocouples placed in the water to guarantee sufficient mixing. The electrical feedthroughs on the collector were connected to Kepco DC power supply and a logging multimeter was placed in series to record the current.

\section{Results and Discussion}

CNT sheets' optical, thermal and electrical conductivity properties are dependent on the reaction temperature and density of the forest from which they are spun. Therefore, to find the best CNT solar selective coating, CNTs with different sheet areal densities were grown by varying the synthesis temperature from 700 to $750^{\circ} \mathrm{C}$ while maintaining the reaction time at 10 minutes. To ensure CNT forest reproducibility, synthesis gas flows, position of the $\mathrm{Si} / \mathrm{Fe}$ substrate inside the reaction chamber, ramping and soaking times were unaltered, and only the temperature was altered. Forest synthesized at a fixed temperature had little variation in height and sheet areal density, Figure 2. However, for different synthesis temperatures, major changes in forest height, sheet areal density, absorption, sheet resistance, forest and sheet morphologies were observed, Table I. Yet, all CNT forests maintained their spinnability over the range of the experimental temperatures. 


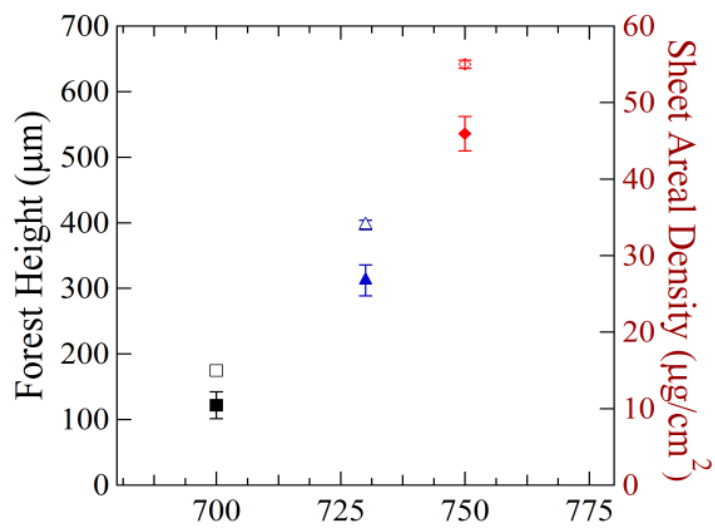

CVD Synthesis Temperature $\left({ }^{\circ} \mathrm{C}\right)$

Figure 2. Characteristic of carbon nanotube forests grown at various synthesis temperatures. Close markers, effect of synthesis temperature on CNT forest height. Open markers, effect of synthesis temperature on Sheet Areal Density.

SEM imaging of the 3 representative forests grown at 700,730 and $750^{\circ} \mathrm{C}$ revealed a change in CNT morphology, straight to wavy, as the temperature increased to $750^{\circ} \mathrm{C}$, Figure $3 \mathrm{a}-$ i. Sheets pulled from the side of these forests align along the CNT pulling direction with thicker bundles and maintain the forest's intrinsic morphology. CNT forest wavy morphology from bottom to top, was observed in samples grown at $730^{\circ} \mathrm{C}$ and $750^{\circ} \mathrm{C}$, but not for forest grown at $700^{\circ} \mathrm{C}$. However, waviness was more pronounced in the forest grown at $750^{\circ} \mathrm{C}$, Figure $3 \mathrm{i}$. Changes in the reaction temperatures affected the diameter of individual CNTs. TEM microscopy revealed that the diameter of the individual CNTs changed from 5 to $10 \mathrm{~m}$ as the temperature increased from 700 to $750^{\circ} \mathrm{C}$, Figure $3 \mathrm{~b}, \mathrm{e}, \mathrm{h}$. 

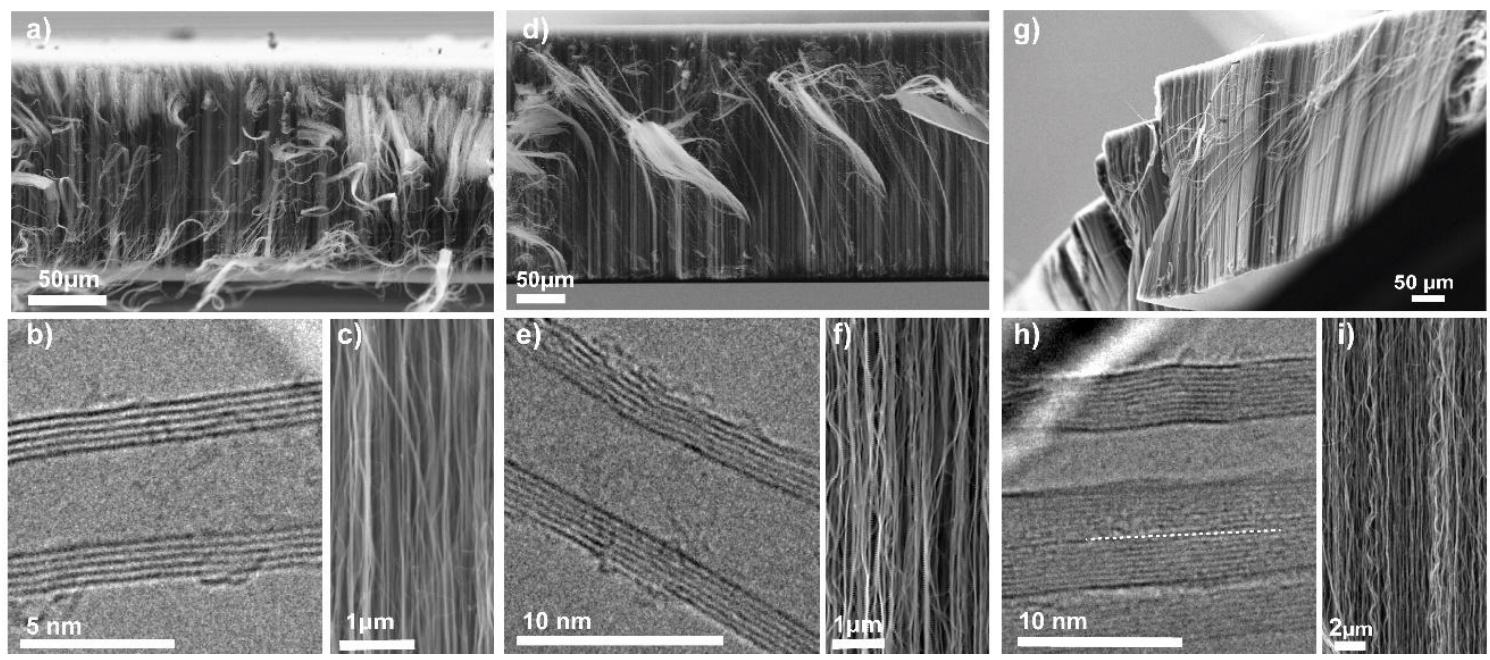

Figure 3. a-c) $\mathrm{CNT}$ forests grown at $700^{\circ} \mathrm{C}$ had heights of $\sim 100 \mu \mathrm{m}$, individual CNT diameters of $\sim 5 \mathrm{~nm}, 4 \mathrm{CNT}$ walls and straight CNTs morphology. d-f) Increased reaction temperature of $730^{\circ} \mathrm{C}$ produced CNT forests with height of $\sim 300 \mu \mathrm{m}$, tube diameters of $\sim 7 \mathrm{~nm}$ with $5 \mathrm{CNT}$ walls and exhibited a semi-wavy morphology, respectively. g-i) Reaction temperature of $750^{\circ} \mathrm{C}$ produced forests with height of $\sim 500 \mu \mathrm{m}$, individual CNT diameters of $\sim 10 \mathrm{~nm}$ and 10 walls. CNT waviness is more pronounced in this type of forests.

At the lowest reaction temperature, $700^{\circ} \mathrm{C}, \mathrm{CNT}$ forests had lengths of $\sim 100 \mu \mathrm{m}$, sheets spun from these forests appeared highly transparent, had low sheet areal density $\left(1.1 \mu \mathrm{g} / \mathrm{cm}^{2}\right)$ and spinnability of 3 (highly spinnable: rating 5, poorly spinnable: rating 1 ). As the reaction temperature increased to $750^{\circ} \mathrm{C}, \mathrm{CNT}$ forests reached heights of $500 \mu \mathrm{m}$, spinnability increased to 5 , sheets spun from these forests had higher sheet areal density $\left(5.6 \mu \mathrm{g} / \mathrm{cm}^{2}\right)$ and were less transparent. At temperatures, higher than $750^{\circ} \mathrm{C}$, forest spinnability sharply decreased and continuous sheets could not be drawn easily. 
Table I. CNT forest and sheet properties. All values are an average of 10 samples.

\begin{tabular}{|c|c|c|c|c|c|c|}
\hline $\begin{array}{c}\text { Temperature } \\
\left({ }^{\circ} \mathbf{C}\right)\end{array}$ & $\begin{array}{l}\text { Time } \\
(\mathbf{m i n})\end{array}$ & $\begin{array}{c}\text { Forest } \\
\text { average } \\
\text { height }(\mu \mathrm{m})\end{array}$ & $\begin{array}{l}\text { Spinnability } \\
\text { rating (0-5) }\end{array}$ & $\begin{array}{c}\text { Sheet areal } \\
\text { density }\left(\mu \mathrm{g} / \mathrm{cm}^{2}\right)\end{array}$ & Sheet $\frac{I_{D}}{I_{C}}$ Ratio & $\begin{array}{c}\text { Sheet } \\
\text { Resistance } \\
(\Omega / \square)\end{array}$ \\
\hline 700 & 10 & 100 & 3 & 1.1 & 0.76 & 950 \\
\hline 730 & 10 & 300 & 4.5 & 3.3 & 0.86 & 548 \\
\hline 750 & 10 & 500 & 5 & 5.6 & 0.96 & 320 \\
\hline
\end{tabular}

Based on the obtained results, CNT sheets spun from the $500 \mu \mathrm{m}$ tall forest produced CNT sheets with the highest areal densities, ideal for solar selective coatings. Thus, the information presented in this study is for those CNT sheets, unless otherwise noted.

\subsection{Optical Properties}

Figure 4 shows UV-Vis measurements, from 300 to $1200 \mathrm{~nm}$, of a quartz slide coated with 5, 8, 10 and $15 \mathrm{CNT}$ sheets spun from the $500 \mu \mathrm{m}$ tall forest. Since these CNT sheets exhibited very low reflection over the visible spectrum, only 5 undensified CNT layers, with sheet areal density of $25 \mu \mathrm{g} / \mathrm{cm}^{2}$, were needed to achieve $<3 \%$ reflection. Reflectance saturated at $\sim 2 \%$ when more than $5 \mathrm{CNT}$ sheets were added, specifically when the sheet areal density was $>25 \mu \mathrm{g} / \mathrm{cm}^{2}$. Beyond 10 layers, the reflectance was not affected by the increase in the CNT sheet areal density, Figure 4a. Light transmission, however, was highly dependent on the amount of CNT sheets added and by the CNT sheet areal density, Figure 4b. 5 undensified sheets showed high transmittance over the solar spectrum and additional layers were needed to approach the saturation value of $\sim 2 \%$. $10 \mathrm{CNT}$ layers, with sheet areal density of $50 \mu \mathrm{g} / \mathrm{cm}^{2}$, were found to achieve the desired saturation value. 

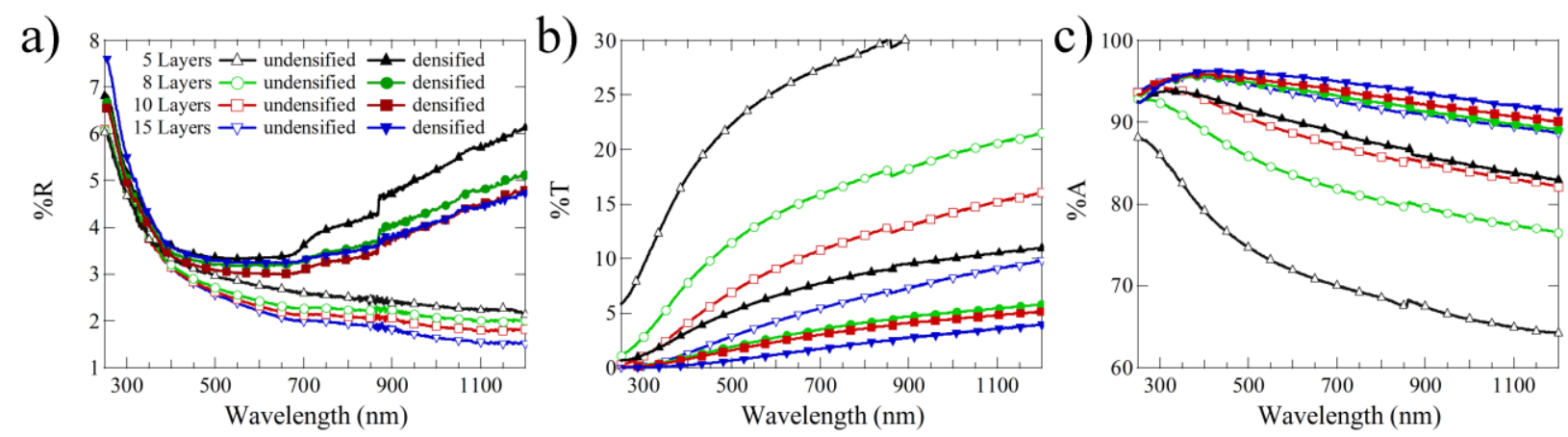

Figure 4. Optical characterization of stacked CNT sheets. a) Reflection, b) transmission, and c) absorption of undensified (open markers) and densified (solid markers) CNT sheets of varying thickness. Densified sheets result in slightly increased reflection as compared to undensified sheets, but this is offset by the significant increase in the absorption of densified sheets. Approximately 10 layers of densified CNTs are sufficient to achieve high absorption.

CNT sheet densification with isopropyl alcohol (IPA) significantly decreased the light transmission, while also slightly increasing the reflection. When densified, surface roughness and thickness of CNT coating decreased due to individual CNTs and bundles collapsing on top of one another, (closing the gaps in the CNT aerogel that allowed the transmission of light), and adopting a planarized morphology. The result is a smooth CNT coating that blocked the transmission of light in an efficient way, but due to the smoother surface, the reflection was compromised. Yet, the overall effect of densification was an increase in the CNT absorption, Figure 4c. Thus, CNT coating spun from the forest grown at $750^{\circ} \mathrm{C}$ with 10 densified layers and areal density of $\sim 50 \mu \mathrm{g} / \mathrm{cm}^{2}$ was determined to be the optimal solar absorber.

The final thickness of the coating was $\sim 3 \mu \mathrm{m}$ and demonstrated the characteristic CNT wavy morphology of the forest, Figure $5 \mathrm{a}-\mathrm{b}$. Sheets spun from this forest achieved the highest absorption, due to photon trapping effects, while having the waviest morphology with respect to 
the other forests. Thus, absorption was found to be independent of the wavy morphology of the CNTs. Changes in the CVD growth parameters will lead to variations in the observed CNT sheet properties. Nevertheless, the overall trend in reflection and transmission should hold.
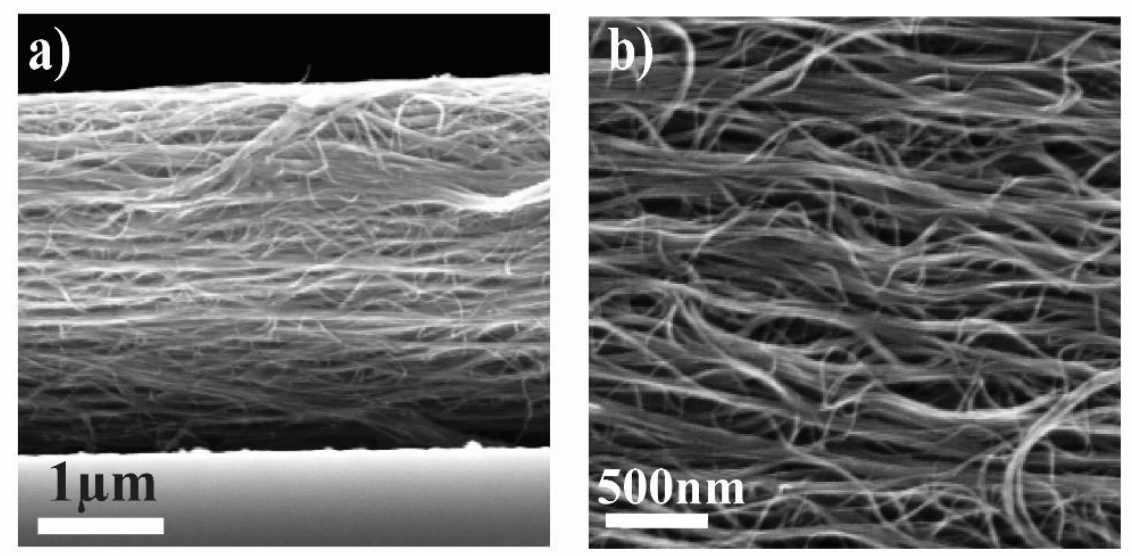

Figure 5. a) Cross-section of the CNT coating on top of Si substrate. The coating was made with 10 layers of CNT sheets densified with IPA. The final areal density was $52 \mu \mathrm{g} / \mathrm{cm}^{2}$ and thickness of $\sim 3 \mu \mathrm{m}$. b) Top view of the wavy morphology of the CNT coating.

Since commercially available SSCs have a semi-transparent selective coating and the collector structure is designated to trap any transmitted light, the reflection of the solar selective coating is an important metric when comparing them to CNT coatings. Figure 6 compares the visible spectrum reflection of the CNT coating to commercial SSC made with sputtered Al-N$\mathrm{Cu} / \mathrm{Al}$ and AL-N SS. The results demonstrated that CNTs outperform the commercial coatings with a steady reflection in the visible spectrum of nearly $2 \%$ and $4 \%$ for the undensified and densified coatings, respectively. By comparison, the best performing commercial coating reflected nearly $9 \%$ with large losses in the low-wavelength part of the spectrum. Since the CNT coatings have low reflection in the visible and near UV region, it is possible to use them as SSCs 
even in an overcast day when the amount of visible radiation is low, but the UV is not greatly affected.

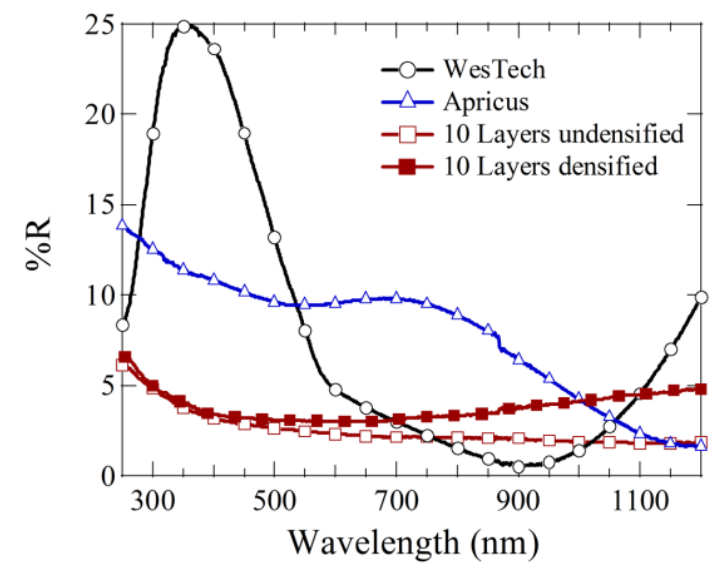

Figure 6. Comparison of reflection between CNT coatings and commercially available SSCs from Apricus (Al-N-Cu/Al) and WesTech (AL-N SS). CNT coatings outperform commercial coatings.

\subsection{Thermal testing}

To test the thermal properties of the CNT coatings, small prototype collectors were prepared by laminating the sheets on top of small glass test tubes, as described in the experimental section. The heating rate and the temperature increase, $\Delta T$, for densified and undensified CNT coatings were recorded inside a high vacuum system. It was found that densified CNT coatings had the highest final temperature, as compared to non-densified, Figure 7a. This difference cannot be explained by optical properties alone, because densified CNT sheets have slightly higher reflectivity and, therein, absorb less energy than the non-densified sheets. Improved thermal contact between densified CNT coating and glass would manifest as increased heating rate of the coating, but no significant difference was observed between the 
densified and undensified sheets. Emissivity, therefore, plays a big role in the performance of the CNT coatings in order to account for this difference in performance.
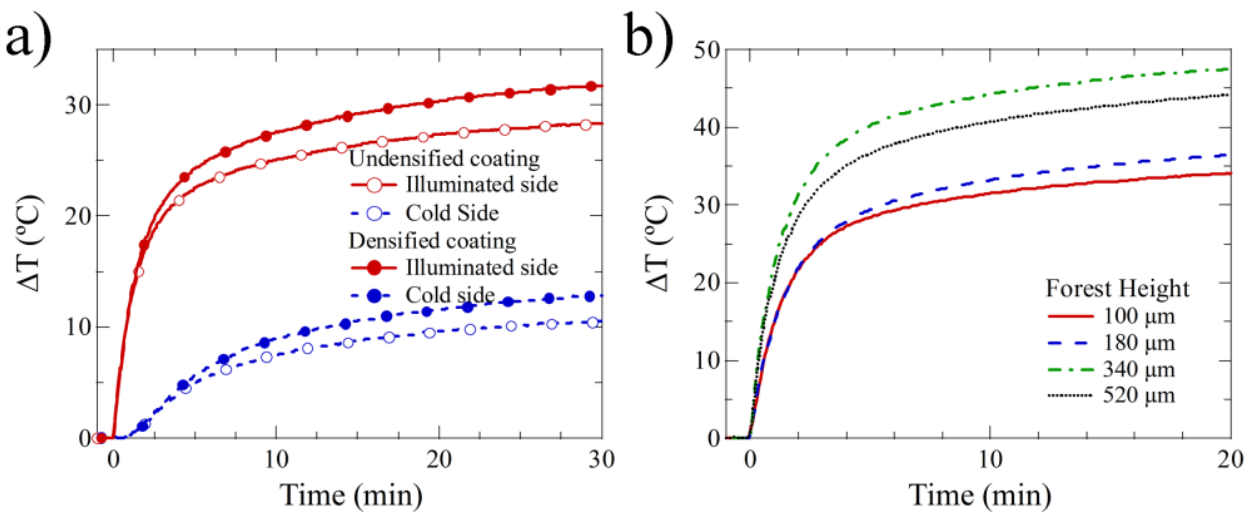

Figure 7. Evaluation of CNT solar selective coating in prototype system under AM 1.5 illumination. a) Typical measurements for CNT coating. After non-densified coating was measured, it was densified with isopropyl alcohol and measured again. b) Measurements of prototype carbon nanotube coatings based on forests with various heights.

Emissivity of densified and undensified CNT coatings was obtained by measuring the reflectivity in the infrared spectrum. Since densification of the CNT coating increased the reflectivity, per Kirchhoff's corollary law for opaque materials, $\varepsilon=1-r$, the emissivity should decrease accordingly. Upon densification, the surface tension effect during the evaporation of the isopropyl alcohol collapsed the CNT sheets and increased the CNT bulk density causing the surface radiation from the interior of the coating to be screened by the outermost layer. This effect caused the emissivity of densified CNT coatings to decrease, which by Kirchhoff's law is represented by an increase in reflectivity, Figure 8.

The emissivity of the densified coating was found to be 0.7 , which constitutes a large energy loss with increasing temperature. In order for CNT coatings to compete with commercially available coatings, the emissivity has to be reduced to below 0.1 . This reduction 
can be accomplished by stacking densified CNT layers with density graded composite sol-gel oxide, such as zinc oxide, deposited on top. Sol-gel oxides are used as electron transporting layers in organic photovoltaics and as n-type semiconductors and are becoming widely and inexpensively available [43]. The ease of processing makes sol-gel oxide an ideal candidate for emissivity suppression. To achieve desired optical properties of superior solar selective coating, optical stack modeling needs to be performed. Optical thicknesses and refractive indices of various layers need to be determined and used in the method of characteristic matrices to simulate and determine optimal composite structure. However, such undertaking is beyond the scope of the present work.

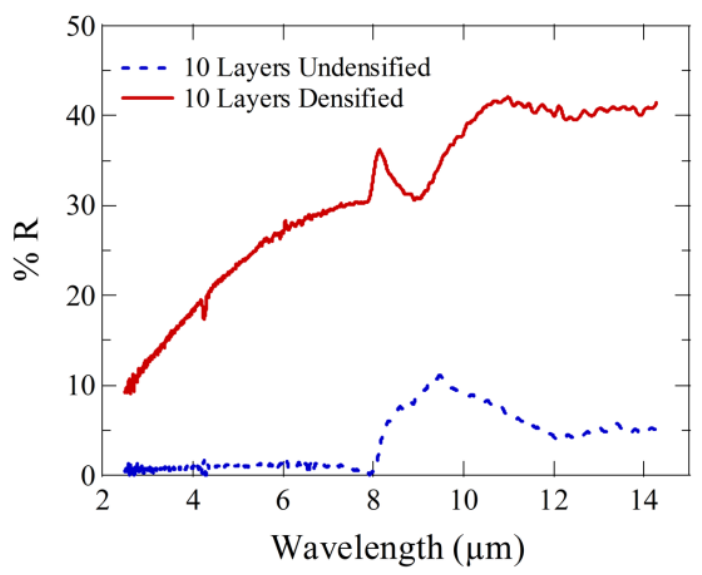

Figure 8. Reflectivity of 10 layers of densified and undensified CNT sheets in the infrared region. Densified CNT coating shows higher reflectance than undensified and by Kirchhoff's law has lower emissivity.

Thermal testing of 10 CNT sheets spun from forests grown at different temperatures, did not reveal significant difference in the heating rates. However, there was a significant difference in the maximum achieved temperature, Figure $7 \mathrm{~b}$. The highest temperature was obtained from CNT coatings spun from forests grown at $730^{\circ} \mathrm{C}$, followed by those grown at $750^{\circ} \mathrm{C}$ and lastly 
those grown at $700^{\circ} \mathrm{C}$. According to Aliev et al. (2009), the thermal conductivity of CNT sheets declines when the forest height, from which they are spun, decreases. As the length of individual CNTs decreases, the number of CNT to CNT bundles per unit length of pulled sheet increases, leading to a suppression of phonon modes. Thus, a reduction in thermal transport is observed [44]. This trend was observed for coatings spun from CNT forest with heights between 100 and $300 \mu \mathrm{m}$ and not for the coatings spun from the $500 \mu \mathrm{m}$ forest. Since the probability of defects occurring in CNT increases with increasing forest synthesis temperature, taller forests, synthesized at $750^{\circ} \mathrm{C}$, are expected to have more intrinsic defects than shorter forest due to its intrinsic wavy morphology. As such, CNT sheets spun from this forest are expected to have lower thermal transport [23,45-47]. Thus, the low thermal transport of the CNT coating spun from the $500 \mu \mathrm{m}$ tall forest can be explained by looking at its morphology. SEM imaging of the $500 \mu \mathrm{m}$ forest revealed a wavy morphology, characteristic of nanotubes grown at high synthesis temperatures [23] with CNT bundle diameter of $\sim 50 \mathrm{~nm}$. The sheets pulled from this forest maintained the forest's intrinsic waviness and aligned along the CNT pulling direction with thicker bundles [23], Figure 9 a-b. To explore the relationship between waviness and CNT defects, Raman analysis of the sheets was performed.
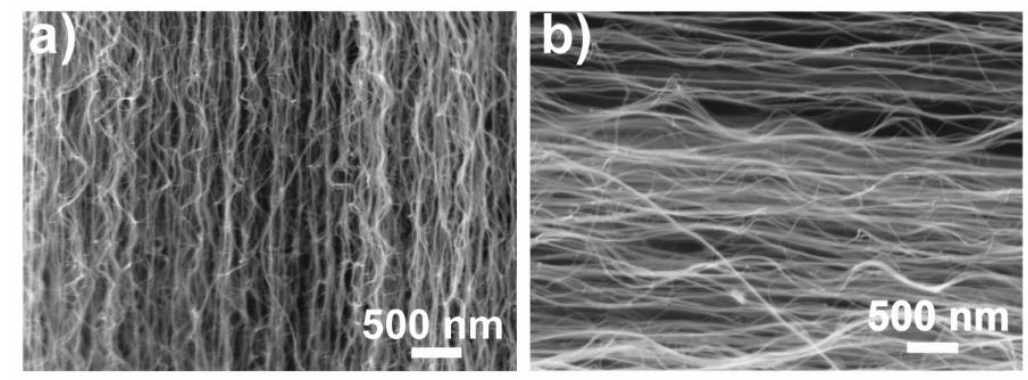

Figure 9. SEM images showing the wavy morphology of the a) CNT forest and b) CNT sheet spun from the $500 \mu \mathrm{m}$ tall forest. 
By taking the ratio of disordered peak, $\mathrm{D}\left(\sim 1350 \mathrm{~cm}^{-1}\right)$, and crystalline graphite peak, $\mathrm{G}$ $\left(\sim 1600 \mathrm{~cm}^{-1}\right)$, the information about crystalline defects and amorphous carbon presented in the CNTs can be obtained [48-50]. In general, smaller $\frac{I_{D}}{I_{G}}$ ratios, are characteristic of samples with high CNT purity and less defects[49]. Therefore, to compare how the temperature and thus, the height, affected the $\frac{I_{D}}{I_{G}}$ ratios of the sheets, CNT spun from the $100-500 \mu \mathrm{m}$ tall forests were recorded, Figure 10. CNT sheets spun from the tallest forest, $500 \mu \mathrm{m}$ exhibited an $\frac{I_{D}}{I_{G}}$ ratio of 0.96, higher than for sheet spun from the medium $\left(\frac{I_{D}}{I_{G}}=0.86\right)$ and short $\left(\frac{I_{D}}{I_{G}}=0.76\right)$ height forests. According to Lee et al. (2013), straighter CNT have lower $\frac{I_{D}}{I_{G}}$ values than wavy CNT and have less defects. Thus, CNT sheets spun from the $500 \mu \mathrm{m}$ tall forests, with the waviest morphology, had $\sim 10 \%$ more crystalline defects per unit length and contained more impurities, i.e., $\mathrm{sp}^{3}$ carbon, than sheets coming from medium and low height forests. Since the highest amount of impurities was found in sheets spun from the $500 \mu \mathrm{m}$ forest, SSC made with this forest had higher phonon scattering that lowered the thermal transport and did not allow the coating to achieve the highest possible temperature increase.

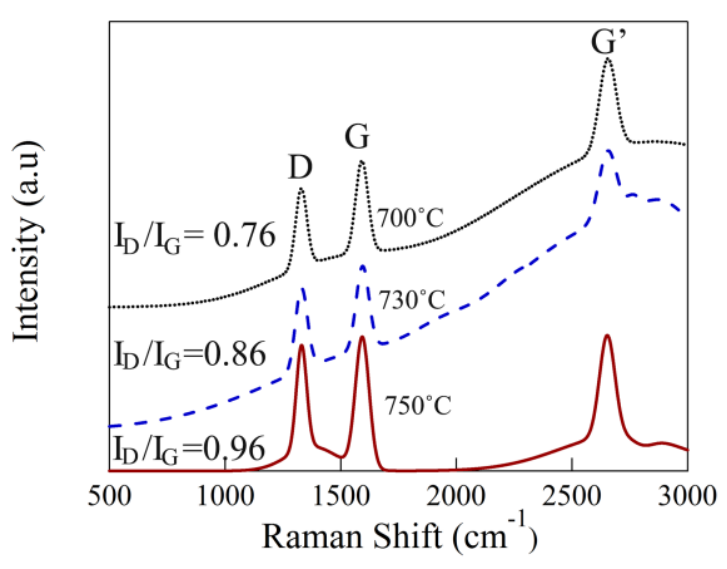


Figure 10. Raman shift of the three types of CNT sheets used as SSC produced at different synthesis temperatures. CNT sheets spun from forests grown at $750^{\circ} \mathrm{C}$ had the highest $\frac{I_{D}}{I_{G}}$ ratio followed by those spun from forest grown at $730^{\circ} \mathrm{C}$. The lowest ratio was recorded for sheets coming from forest synthesized at $700^{\circ} \mathrm{C}$.

In general, CNT-SSCs must have good thermal conductivity, low thermal contact resistance between the glass and CNT coating, high absorption and low reflection in the visible range. Low emissivity in the IR (corresponding to the black body emission peak of heated ETC inner tube at operational temperature) is also desirable. Optical measurements provide a guideline for the implementation of CNT sheets as solar selective coatings; however, the performance is a convolution of all the properties. To that end, CNT growth parameters, such as gas flow, reaction temperature and time, need to be further optimized to produce spinnable forest with long CNTs from which SSCs can be spun with minimal defects.

\subsection{Joule heating}

Slow heating rate and the dependence on solar energy are major drawbacks of solar water heaters, particularly on a cloudy or overcast day. In the real world, SWH systems generate hot water after several hours of exposure to solar energy, i.e. around noon, which is after the morning peak consumption of hot water (at $7-9$ am [41]). Since the heating rate of water in standard ETC systems is slow, there are several ways to provide hot water in the late evening hours, when the sun is not available. ETC filled with phase change materials (PCMs), such as erythritol, have been reported to allow the accumulation of heat and prolonged the availability of hot water for 3-4 hours after sunset [51]. Another approach is to incorporate PCM into the CNT 
selective coatings so when the PCM melts, the heat gets accumulated in the outer surface of the ETC inner tube [52].

ETCs rely on booster hardware, (electrical heaters) and the unspent hot water reserves from the previous night, which are subject to large environmental losses over extended periods of time at low ambient temperatures. By utilizing the Joule heating capability of the electrically conductive CNT coating in the ETC would remove the need for booster hardware and through the use of a feedback system allow the solar water heater to operate at the optimal temperature (even with limited solar irradiance on cloudy days) and achieve high efficiency. By Appling current to the CNTs, the coating is heated to heat from absorbed solar light), similarly to the heating elements in a conventional water heaters, and transfers the heat to the water flowing through the collector or the heat pipe. Thus, the Joule heating in CNTs is fast, efficient and combined with the vacuum insulated structure of the evacuated tube collectors produces fast ondemand water heater system.

To test the Joule heating capabilities, the inner tube of an ETC was coated with the CNTSSC and miniature evacuated tube collector was fabricated. The inside of the ETC was directly filled with tap water to eliminate the effect of the heat pipe on the performance. Based on the relationship, Equation 1, between the energy, $E$, the mass of water, $m$, and the specific heat of water, $c$, the instantaneous efficiency was defined in Equation 2, where $E_{D C}$ is the energy and $P_{D C}$ is the power from the electrical power supply.

$$
\begin{gathered}
E=c m \Delta T \\
\eta_{\text {inst }}=\frac{\frac{d}{d t}(c m \Delta T)}{\frac{d}{d t}\left(E_{D C}\right)}=\frac{c m \frac{d \Delta T}{d t}}{P_{D C}}
\end{gathered}
$$

At $95 \mathrm{~W}$ of applied power, the water inside the collector reached temperatures of over $90^{\circ} \mathrm{C}$ in less than 60 minutes. The performance of the system varies with the applied power and 
the temperature difference between the water inside the collector and the environment due to thermal losses. Starting water temperature is a good estimate for the ambient temperature for a residential water heater system. At elevated ambient temperatures, the heated water will reach higher temperatures. Thus, the change of efficiency with increasing water temperature indicates the amount of thermal losses from convection, conduction and re-radiation from the system, Figure 11. This effect is similar to the typical performance of SWH systems under different incident irradiances.

The increase in efficiency with applied power is due to the difference in the heating rate and the heat loss time constants. With higher applied power, the heating time constant is smaller and the large heat loss time constant has smaller contribution to the system's heating efficiency. Therein, Joule heating of CNT coatings achieved $80-90 \%$ water heating efficiency in the residential heating temperature range $\left(\Delta \mathrm{T}=40-60^{\circ} \mathrm{C}\right)$.

The Joule heating of CNT coated ETCs provides fast heating rates capable of generating hot water on-demand at any time of the day, independently of the intensity of solar light and without recirculation. Since the typical sun power reaching an ETC of a standard size is small $90 \mathrm{~W}$ (at AM1 solar radiation), relying only on it provides slow water heating rates in conventional ETC solar water heaters [51,52]. However, for the Joule heating of CNT-ETC, by applying a higher current, the power can be changed and the desired hot water temperature can be achieved in a shorter time. For an ideal fast heating, an overall CNT-ETC resistance of 20-30 $\Omega$ is needed. This can be achieved by laminating $~ 35$ layers of CNT with individual sheet resistance of $100 \Omega / \square$. In this case a current of 3-4 A and a power of 300-480 W (3 to 5 times larger compared to $90 \mathrm{~W}$ power of direct sun), can be readily applied to the CNT-ETC and increase the heating rate significantly. To achieve a low CNT resistance without adding extra 
CNT layers, the authors have proven that the incorporation of graphitic powder between CNT layers during the dry-lamination process is effective [53].

CNT Joule heating functionality could operate similarly to tankless water heaters to provide instantaneous hot water, when needed, quickly and efficiently. With the vacuum insulated structure of the ETCs, low thermal losses are achieved. In this way, SWH with CNT as solar selective coating can function as conventional, slow solar water heater system with a storage tank (when enough solar energy is available) and as instant hot water system, without a need for a separate electrical booster system.

For an early stage prototype, the presented results are very promising for the development of dual power CNT-ETC that can function with direct solar energy or Joule heating. Furthermore, Joule heating and solar heating can be combined with a smart control system to manage daily water usage. Such system will generate the daily required amount of water in the allotted time in the most efficient way through the combination of the two heating systems in a single CNT-ETC collector. With internet connection, the system will be able to anticipate the amount of available solar energy based on the weather forecast and make necessary adjustments to the heating processes to deliver hot water based on the household's individual consumption schedule.
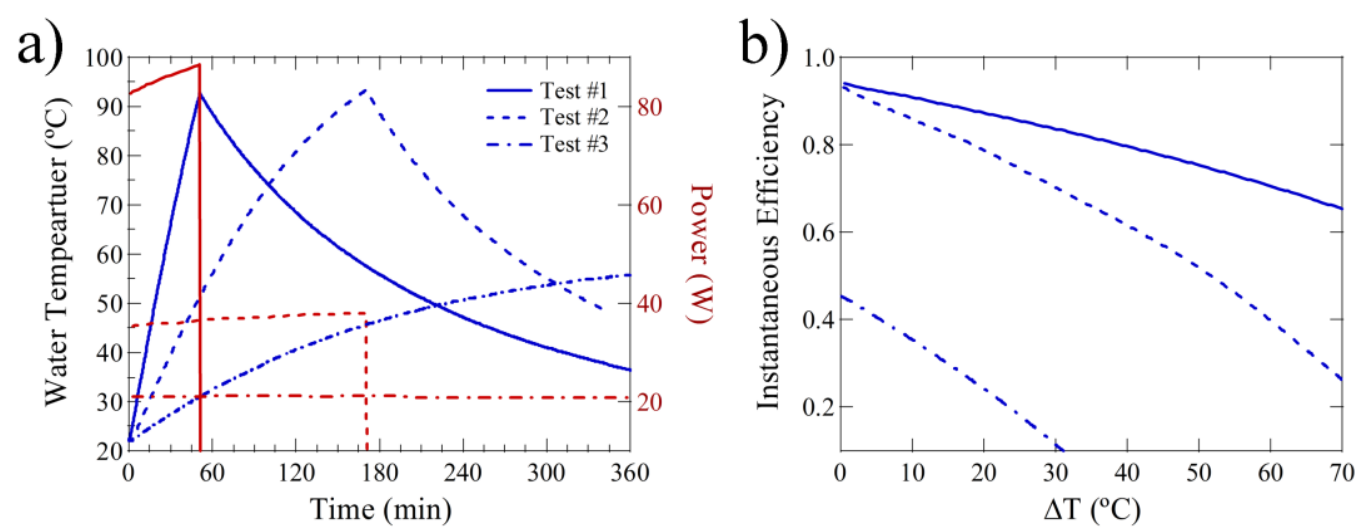
Figure 11. Demonstration of Joule heating functionality of CNT solar selective coatings.

a) Maximum temperature and heating rate dependent on the applied power, (Test \#1- 90W, \#240W and \#3- 20W. b) The efficiency of the CNT solar selective coatings. The system achieves efficiency of $80-90 \%$ in the residential water heater system range.

\section{Conclusions}

In this study, dry-spun CNT coatings obtained from spinnable forests have proven to be suitable solar selective coating for ETC water heaters with a wide operational spectral range.

Optical selectivity was found to be dependent on sheet areal density, CNT diameter and number of CNT walls. CNT coatings with the highest optical absorption, spun from the tallest forest $(500 \mu \mathrm{m})$, had the highest sheet areal density, larger CNT diameter $(\sim 10 \mathrm{~nm})$ and higher number of CNT walls ( 10 walls). Therefore, CNT sheets spun from $500 \mu \mathrm{m}$ tall forest were the best solar absorbers, but were also the waviest and had the highest number of defects per sheet length that constrained the thermal transport. Thus, CNT sheets spun from medium height forest with a straight CNT morphology are favorable for better thermal transport.

CNT coating Joule heating capability is a fast and efficient way to electrically heat water that cannot be achieved by the state of the art selective coatings since they are, almost always, electrical insulators. Thus, by reducing the emissivity of the CNT coating with densification and optimizing the joule heating power output, the efficiency of the CNT-ETC can be further improved.

\section{Acknowledgements}

This work was supported by the U.S. Department of Energy (DOE) under the STTR award DE-SC0009447. P.M. would like to acknowledge CONACYT and the Mexican government for academic opportunities and support. The authors would like to thank Apricus Inc. for the donation of the evacuated tube collectors, Dr. Mick Humphrey of Apricus for 
numerous helpful discussions on selective coatings and Juan Pablo Oviedo for the help with TEM images. Partial financial support from the Ministry of Education and Science of the Russian Federation (Grant № 14.Y26.31.0010 for optical measurements) and grant in the framework of Increase Competitiveness Program of NUST "MISiS" (No. K2-2015-014 for samples preparation) is highly appreciated. We also appreciate the partial support of Welch Foundation grant AT 16-17.

\section{References}

[1] International Energy Agency IEA, Solar Energy Perspectives, 2011. doi:10.1787/9789264124585-en.

[2] International Energy Agency (IEA), Key World Energy Statistics 2016, (2016).

[3] Y. Tian, C.Y. Zhao, A review of solar collectors and thermal energy storage in solar thermal applications, Appl. Energy. 104 (2013) 538-553.

doi:10.1016/j.apenergy.2012.11.051.

[4] C.E. Kennedy, Review of Mid- to High- Temperature Solar Selective Absorber Materials Review of Mid- to High- Temperature Solar Selective Absorber Materials, (2002).

[5] O. Ilic, P. Bermel, G. Chen, J.D. Joannopoulos, I. Celanovic, M. Soljačić, Tailoring hightemperature radiation and the resurrection of the incandescent source, (2016). doi:10.1038/NNANO.2015.309.

[6] J.B. Chou, Y.X. Yeng, Y.E. Lee, A. Lenert, V. Rinnerbauer, I. Celanovic, M. Soljačić, N.X. Fang, E.N. Wang, S.G. Kim, Enabling ideal selective solar absorption with 2D metallic dielectric photonic crystals, Adv. Mater. 26 (2014) 8041-8045. doi:10.1002/adma.201403302.

[7] N. Selvakumar, H.C. Barshilia, Review of physical vapor deposited (PVD) spectrally 
selective coatings for mid- and high-temperature solar thermal applications, Sol. Energy Mater. Sol. Cells. 98 (2012) 1-23. doi:10.1016/j.solmat.2011.10.028.

[8] H. Kataura, Y. Kumazawa, Y. Maniwa, I. Umezu, S. Suzuki, Y. Ohtsuka, Y. Achiba, Optical properties of single-wall carbon nanotubes, Synth. Met. 103 (1999) 2555-2558. doi:10.1016/S0379-6779(98)00278-1.

[9] L.A. Falkovsky, Optical properties of graphene, J. Phys. Conf. Ser. 129 (2008) 12004. doi:10.1088/1742-6596/129/1/012004.

[10] A.E. Aliev, M.H. Lima, E.M. Silverman, R.H. Baughman, Thermal conductivity of multiwalled carbon nanotube sheets: radiation losses and quenching of phonon modes, Nanotechnology. 21 (2010). doi:10.1088/0957-4484/21/3/035709.

[11] Y. Ito, Y. Tanabe, J. Han, T. Fujita, K. Tanigaki, M. Chen, Multifunctional Porous Graphene for High-Efficiency Steam Generation by Heat Localization, Adv. Mater. 27 (2015) 4302-4307. doi:10.1002/adma.201501832.

[12] J. Bernholc, D. Brenner, M.B. Nardelli, V. Meunier, C. Roland, MECHANICAL AND ELECTRICAL PROPERTIES OF NANOTUBES, Annu. Rev. Mater. Res. 32 (2002) 347-75. doi:10.1146/annurev.matsci.32.112601.134925.

[13] M. Vakili, S.M. Hosseinalipour, S. Delfani, S. Khosrojerdi, M. Karami, Experimental investigation of graphene nanoplatelets nanofluid-based volumetric solar collector for domestic hot water systems, Sol. Energy. 131 (2016) 119-130. doi:10.1016/j.solener.2016.02.034.

[14] J. Kang, H. Kim, || Keun, S. Kim, S.-K. Lee, S. Bae, J.-H. Ahn, Y.-J. Kim, J.-B. Choi, B.H. Hong, High-Performance Graphene-Based Transparent Flexible Heaters, (2011). doi:10.1021/nl202311v. 
[15] Z.-P. Yang, L. Ci, J.A. Bur, S.-Y. Lin, P.M. Ajayan, Experimental Observation of an Extremely Dark Material Made By a Low-Density Nanotube Array, (n.d.). doi:10.1021/n1072369t.

[16] Z. Chen, T. Boström, Electrophoretically deposited carbon nanotube spectrally selective solar absorbers, Sol. Energy Mater. Sol. Cells. 144 (2016) 678-683. doi:10.1016/j.solmat.2015.10.016.

[17] A. Cao, X. Zhang, C. Xu, B. Wei, D. Wu, Tandem structure of aligned carbon nanotubes on Au and its solar thermal absorption, Sol. Energy Mater. Sol. Cells. 70 (2002) 481-486. doi:10.1016/S0927-0248(01)00083-6.

[18] N. Selvakumar, S.B. Krupanidhi, H.C. Barshilia, Carbon Nanotube-Based Tandem Absorber with Tunable Spectral Selectivity: Transition from Near-Perfect Blackbody Absorber to Solar Selective Absorber, Adv. Mater. 26 (2014) 2552-2557. doi:10.1002/adma.201305070.

[19] M. Zhang, S. Fang, A.A. Zakhidov, S.B. Lee, A.E. Aliev, C.D. Williams, K.R. Atkinson, R.H. Baughman, Strong, Transparent, Multifunctional, Carbon Nanotube Sheets, Science (80-. ). 309 (2005).

[20] R. Saito, G. Dresselhaus, M. Dresselhaus, Physical Properties of Carbon Nanotubes, Imperial College Press, 1998.

[21] M. Kumar, Y. Ando, Chemical vapor deposition of carbon nanotubes: a review on growth mechanism and mass production., J. Nanosci. Nanotechnol. 10 (2010) 3739-3758. doi:10.1166/jnn.2010.2939.

[22] R.D. Bennett, A.J. Hart, R.E. Cohen, Controlling the morphology of carbon nanotube films by varying the areal density of catalyst nanoclusters using block-copolymer micellar 
thin films, Adv. Mater. 18 (2006) 2274-2279. doi:10.1002/adma.200600975.

[23] C.P. Huynh, S.C. Hawkins, Understanding the synthesis of directly spinnable carbon nanotube forests, Carbon N. Y. 48 (2010) 1105-1115. doi:10.1016/j.carbon.2009.11.032.

[24] V. Sivakumar, A.R. Mohamed, A.Z. Abdullah, S.P. Chai, Role of reaction and factors of carbon nanotubes growth in chemical vapour decomposition process using Methane-A highlight, J. Nanomater. 2010 (2010) 1-11. doi:10.1155/2010/395191.

[25] A. Eatemadi, H. Daraee, H. Karimkhanloo, M. Kouhi, N. Zarghami, A. Akbarzadeh, M. Abasi, Y. Hanifehpour, S.W. Joo, Carbon nanotubes: properties, synthesis, purification, and medical applications., Nanoscale Res. Lett. 9 (2014) 393. doi:10.1186/1556-276X-9393.

[26] B. Hornbostel, M. Haluska, J. Cech, U. Dettlaff, S. Roth, Arc Discharge and Laser Ablation Synthesis of Singlewalled Carbon Nanotubes, Carbon Nanotub. 1 (2006) 1-18. doi:10.1007/1-4020-4574-3_1.

[27] N. Arora, N.N. Sharma, Arc discharge synthesis of carbon nanotubes: Comprehensive review, Diam. Relat. Mater. 50 (2014) 135-150. doi:10.1016/j.diamond.2014.10.001.

[28] Y. Ando, S. Iijima, Preparation of Carbon Nanotubes by Arc-Discharge Evaporation, Jpn. J. Appl. Phys. 32 (1993) L107-L109. doi:10.1143/JJAP.32.L107.

[29] G.D. Nessim, A.J. Hart, J.S. Kim, D. Acquaviva, J. Oh, C.D. Morgan, M. Seita, J.S. Leib, C. V Thompson, Tuning of Vertically-Aligned Carbon Nanotube Diameter and Areal Density through Catalyst Pre-Treatment, (n.d.). doi:10.1021/nl801437c.

[30] H. Kim, W. Sigmund, Iron particles in carbon nanotubes, Carbon N. Y. 43 (2005) 17431748. doi:10.1016/j.carbon.2005.02.019.

[31] H. Yoshida, S. Takeda, T. Uchiyama, H. Kohno, Y. Homma, Atomic-Scale In-situ 
Observation of Carbon Nanotube Growth from Solid State Iron Carbide Nanoparticles, (n.d.). doi:10.1021/n1080452q.

[32] C. Brucker, T. Rhodin, Chemisorption and reaction of acetylene and ethylene on the $\alpha$ Fe(100) clean iron surface, J. Catal. 47 (1977) 214-231. doi:10.1016/00219517(77)90169-5.

[33] X. Lepró, M. Rcio, D. Lima, R.H. Baughman, Spinnable carbon nanotube forests grown on thin, flexible metallic substrates, (2010). doi:10.1016/j.carbon.2010.06.016.

[34] Z. Zang, A. Nakamura, J. Temmyo, Single cuprous oxide films synthesized by radical oxidation at low temperature for PV application, Opt. Express. 21 (2013) 4188-4192. doi:10.1364/OE.21.011448.

[35] S. Eisermann, A. Kronenberger, A. Laufer, J. Bieber, G. Haas, S. Lautenschläger, G. Homm, P.J. Klar, B.K. Meyer, Copper oxide thin films by chemical vapor deposition: Synthesis, characterization and electrical properties, Phys. Status Solidi. 209 (2012) 531536. doi:10.1002/pssa.201127493.

[36] G.L. Morrison, I. Budihardjo, M. Behnia, Water-in-glass evacuated tube solar water heaters, Sol. Energy. 76 (2004) 135-140. doi:10.1016/j.solener.2003.07.024.

[37] Z. Pluta, Evacuated tubular or classical flat plate solar collectors?, Open Access J. J. Power Technol. 91 (2011) 158-164.

[38] C.C. Smith, T.A. Weiss, Design application of the Hottel-Whillier-Bliss equation, Sol. Energy. 19 (1977) 109-113. doi:10.1016/0038-092X(77)90047-0.

[39] M.J. Ahmad, G.N. Tiwari, Optimization of Tilt Angle for Solar Collector to Receive Maximum Radiation, Open Renew. Energy J. 2 (2009) 19-24.

[40] M.J. Carvalho, P. Horta, J.F. Mendes, M.C. Pereira, W.M. Carbajal, Incidence Angle 
Modifiers: A General Approach for Energy Calculations, in: Proc. ISES World Congr. 2007 (Vol. I - Vol. V), Springer Berlin Heidelberg, Berlin, Heidelberg, 2008: pp. 608612. doi:10.1007/978-3-540-75997-3_112.

[41] V. Dabra, L. Yadav, A. Yadav, The effect of tilt angle on the performance of evacuated tube solar air collector: experimental analysis, Int. J. Eng. Sci. Technol. 5 (2013) 100-110.

[42] E. Zambolin, D. Del Col, Experimental analysis of thermal performance of flat plate and evacuated tube solar collectors in stationary standard and daily conditions, Sol. Energy. 84 (2010) 1382-1396. doi:10.1016/j.solener.2010.04.020.

[43] X. Yu, T.J. Marks, A. Facchetti, Metal oxides for optoelectronic applications, Nat. Mater. 15 (2016) 383-396. doi:10.1038/nmat4599.

[44] A.E. Aliev, C. Guthy, M. Zhang, S. Fang, A.A. Zakhidov, J.E. Fischer, R.H. Baughman, Thermal transport in MWCNT sheets and yarns, Carbon N. Y. 45 (2007) 2880-2888. doi:10.1016/j.carbon.2007.10.010.

[45] A.M. Marconnet, M.A. Panzer, K.E. Goodson, Thermal conduction phenomena in carbon nanotubes and related nanostructured materials, Rev. Mod. Phys. 85 (2013) 1295-1326. doi:10.1103/RevModPhys.85.1295.

[46] W. Lin, J. Shang, W. Gu, C.P. Wong, Parametric study of intrinsic thermal transport in vertically aligned multi-walled carbon nanotubes using a laser flash technique, Carbon N. Y. 50 (2012) 1591-1603. doi:10.1016/j.carbon.2011.11.038.

[47] J. Lee, E. Oh, H.J. Kim, S. Cho, T. Kim, S. Lee, J. Park, H.J. Kim, K.H. Lee, The reason for an upper limit to the height of spinnable carbon nanotube forests, J. Mater. Sci. 48 (2013) 6897-6904. doi:10.1007/s10853-013-7494-3.

[48] B.L. Crossley, N.E. Glauvitz, B.T. Quinton, R.A. Coutu, P.J. Collins, P.J. Collins, 
Characterizing Multi-Walled Carbon Nanotube Synthesis for Field Emission Applications, in: Carbon Nanotub. Appl. Electron Devices, InTech, 2011. doi:10.5772/17782.

[49] M.S. Dresselhaus, A. Jorio, A.G. Souza Filho, A.R. Saito, Defect characterization in graphene and carbon nanotubes using Raman spectroscopy, Phil. Trans. R. Soc. A. 368 (2010) 5355-5377. doi:10.1098/rsta.2010.0213.

[50] R.A. DiLeo, B.J. Landi, R.P. Raffaelle, Purity assessment of multiwalled carbon nanotubes by Raman spectroscopy, J. Appl. Phys. 101 (2007) 64307. doi:10.1063/1.2712152.

[51] A. Papadimitratos, S. Sobhansarbandi, V. Pozdin, A. Zakhidov, F. Hassanipour, Evacuated tube solar collectors integrated with phase change materials, Sol. Energy. 129 (2016) 10-19. doi:10.1016/j.solener.2015.12.040.

[52] S. Sobhansarbandi, P.M. Martinez, A. Papadimitratos, A. Zakhidov, F. Hassanipour, Evacuated tube solar collector with multifunctional absorber layers, Sol. Energy. 146 (2017) 342-350. doi:10.1016/j.solener.2017.02.038.

[53] P.M Martinez, G. Smagulova, G. Prihodko, Z. Mansurov, A. Zakhidov, Selective coatings by co-lamination of CNT sheets with rice husk derived graphitic nanopowders, Eurasian Chem. Tech. Journal, 2017 (in press). 

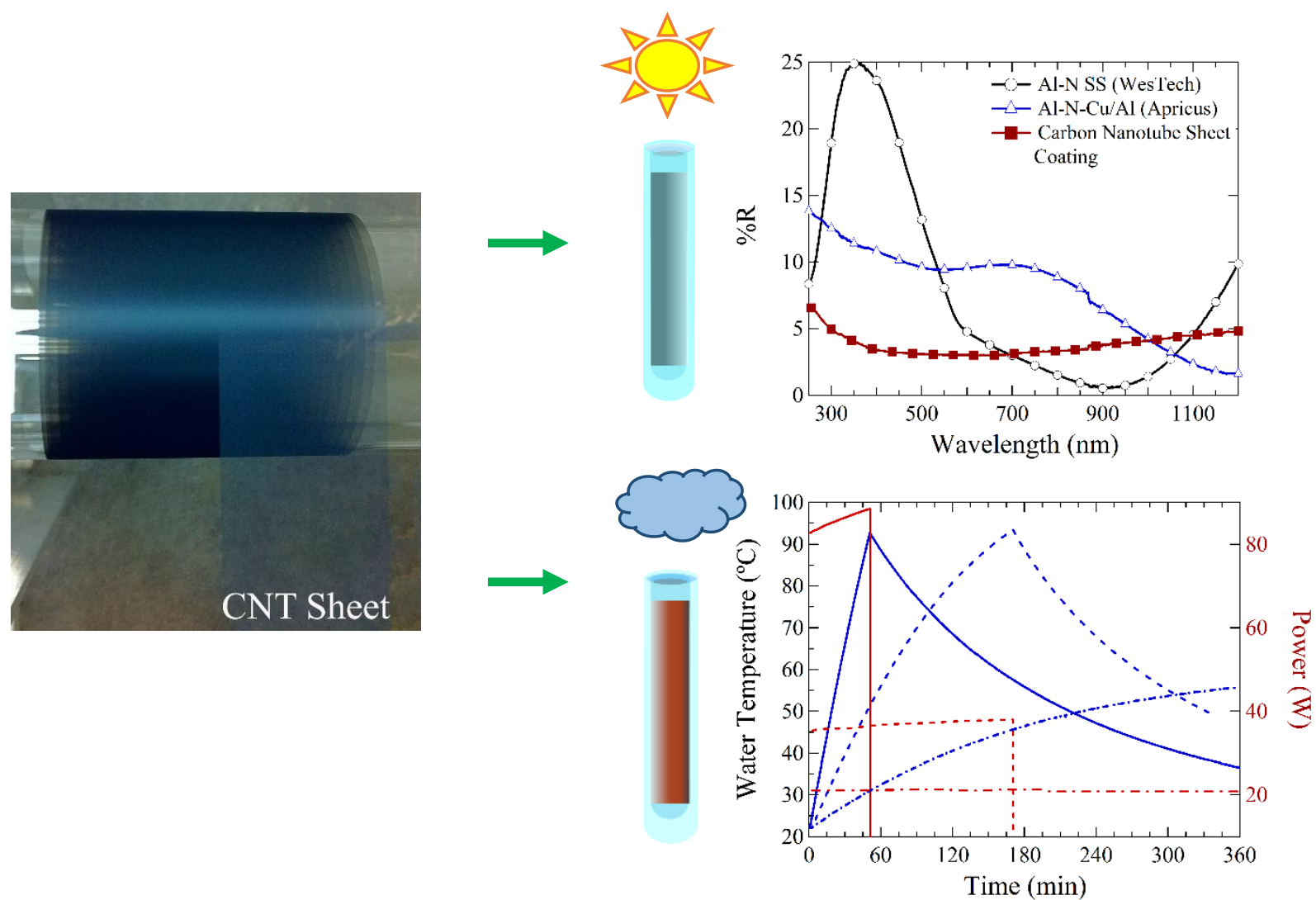